\title{
O CUIDADO DA ENFERMAGEM NO SEGUIMENTO DE CRIANÇAS PRÉ-TERMO E DE BAIXO PESO AO NASCER
}

\author{
THE NURSING CARE TO PRETERM AND LOW BIRTH \\ WEIGHT INFANT IN HOME FOLLOW UP
}

\author{
Débora F. Mello ${ }^{2}$ \\ Semiramis M. M. Rocha ${ }^{2}$ \\ Carmem $^{\wedge}$ I G. S. Scochi ${ }^{2}$ \\ Regina A. G. Lima ${ }^{2}$
}

MELLO, D.F.; ROCHA, S.M.M.; SCOCH^I, C.G.S.; LIMA, R.A.G. O cuidado da enfermagem no seguimento de crianças pré-termo e de baixo peso ao nascer. Reu Bras. Cresc. Desenv. Hum., São Paulo, 10(1), 49-58, 2000.

Resumo: A assistência às crianças pré-termo e de baixo peso ao nascer avançou muito nos últimos anos com o incremento da tecnologia e a queda dos índices de mortalidade infantil, refletindo na sobrevida de um maior número de crianças e desencadeando questionamentos quanto à qualidade de vida, às necessidades de cuidados e ao seguimento a longo prazo. Esta investigação tem como objetivo identificar os cuidados domiciliares prestados a criança pré-termo e de baixo peso ao nascer, caracterizando o seguimento durante o primeiro mês após a alta hospitalar. O enfoque metodológico contempla abordagens da epidemiologia, do cuidado de enfermagem e das políticas de saúde. O estudo empírico do cuidado no domicílio, baseado na consulta a dados em prontuários hospitalares, na observação participante e nos relatos orais das mães, destaca as características das crianças e das mães estudadas, da hospitalização e do vínculo e dos cuidados de saúde infantil no domicílio. A partir dessa configuração, aponta-se aspectos do seguimento dessas crianças através de visitas domiciliares, compondo elementos básicos dos cuidados de enfermagem. O seguimento domiciliar envolve o cuidar da criança em suas necessidades clínicas, na prevenção de riscos e de danos, na alimentação, na higiene, no uso de medicações, nas estimulações sensoriais, entre outros aspectos, e envolve o cuidar da mãe estando ao seu lado, escutando-a, revivendo com ela experiências do processo de hospitalização da criança, cuidando do bebe conjuntamente, proporcionando educação em saúde, conforto e autoconfiança. Ao mesmo tempo, o cuidado no domicílio implica em levantar outras necessidades da família e os recursos da comunidade, integrando informações e intervenções entre os serviços de saúde e a rede de serviços de apoio.

Palavras-chave: pre-terrno; baixo peso ao nascer; seguimento; enfermagem.

\section{INTRODUÇÃO}

Nos últimos anos as investigações em saúde, o incremento da tecnologia e a queda nos índices de mortalidade infantil contribuíram para o avanço da assistência à saúde de recémnascidos. As crianças pré-termo e de baixo peso ao nascer têm sido estudados sob vários enfoques, sendo cada vez mais crescente a preocupação com os fatores de risco perinatais, neonatais e durante toda a infância.

A literatura vem apontando como fatores predisponentes a idade materna (abaixo de 16 anos e acima de 40 anos), primiparidade, adolescentes

1 Este trabalho faz parte do Projeto integrado de Pesquisa "Estudo da Assistência integral à Criança e ao Adolescente", Processo CNPq n 524507/96 - 1 (NV). Baseado na Tese de Doutorado: O cuidado de enfermagem no seguimento de crianças pré-termo e de baixo peso. Escola de Enfermagem de Ribeirão Preto, Universidade de São Paulo, 1998.

2 Docentes do Departamento de Enfermagem Materno-lnfantil e Saúde Pública da Escola de Enfermagem de Ribeirão Preto da Universidade de São Paulo. End. Av. Bandeirantes, 3900 - Ribeirão Preto - s^P - CEP 14040-902.

Correpomdência para: Profa. Dra. Débora Falleiros de Mello. 
solteiras, pequeno intervalo entre as gestações, má nutrição materna, algumas patologias (hipertensão arterial, cardiopatias, diabetes, intercorrências obstétricas, entre outras), ausência de cuidado prénatal, fumo, consumo de álcool e drogas, riscos ocupacionais, baixo nível de escolaridade materna e situação sócio-econômica desfavorável. Muitas causas do nascimento prematuro e do baixo peso ao nascer são desconhecidas. Fatores biológicos já foram evidenciados e, tanto em investigações nacionais como internacionais, têm sido colocada a correlação entre esses fatores, com destaque para a associação entre a incidência da prematuridade e do baixo peso ao nascer e a situação socioeconômica (SCHAFFER \& AVERY, 1979; MONTEIRO et al., 1980; AMON et al., 1987 ALBERMAN \& EVANS, 1992; FUNDAÇÃO IBGE, 1992, AVERY, 1994).

A alta hospitalar de crianças pré-termo e de baixo peso ao nascer não significa a resolução de todos os problemas de saúde, imprimindo a necessidade de acompanhamento ou seguimento dessas crianças a longo prazo. O seguimento é apontado como essencial para se reconhecer a eficácia dos cuidados perinatais, detecção precoce e tratamento de problemas de saúde e prevenção de riscos de morbimortalidade. Os altos investimentos realizados nas unidades neonatais, tanto científicos, tecnológicos e financeiros, como os emocionais, alcançarão uma maior significância se houver um seguimento do crescimento e desenvolvimento global dessas crianças (WELDT et al., 1989; BARBOSA et al., 1993; PENALVA, 1996).

A compreensão da relação entre os fatores de risco a que as crianças estão expostas, as características qualitativas sobre os cuidados à saúde, o aporte da rede de serviços de saúde, as intervenções em visitas domiciliares, pode fornecer à enfermagem e demais profissionais de saúde elementos importante para melhorar o seguimento do crescimento e desenvolvimento infantil e da atenção à saúde das famílias.

Neste estudo, delineamos como objeto central de investigação os cuidados domiciliares no seguimento de crianças pré-termo e de baixo peso ao nascer, tendo por objetivos a identificação dos cuidados domiciliares durante o primeiro mês após a alta hospitalar, através de visitas domiciliares, e a caracterização do seguimento dessas crianças através da observação e do diálogo com a mãe.

\section{ABORDAGEM METOGOLÓGICA}

O referencial teórico desta investigação está fundamentado na pesquisa qualitativa em saúde, que trabalha com os significados das ações, motivações, aspirações, crenças, valores, atitudes e relações humanas, apreendidos a partir do olhar do pesquisador que capta apenas um fragmento ou parte de uma realidade. A ênfase da abordagem qualitativa está em compreender e analisar a dinâmica das relações sociais estabelecidas com a vivência e experiência no cotidiano, compreendidas dentro de estruturas e instituições (MINAYO, 1994).

Neste estudo, para delinear o objeto de investigação, foi importante recorrer ao campo de conhecimentos da Epidemiologia, apontando as relações entre riscos e danos para os problemas de prematuridade e baixo peso ao nascer; também foi relevante buscar relacionar as políticas sociais e de saúde implantadas pelo Estado e, por outro lado, apreender os componentes dos cuidados básicos de enfermagem na assistência à saúde da criança, retratando o seguimento em um trabalho compartilhado com a mãe. A epidemiologia moderna, ao trabalhar com o conceito de risco, coloca que os fatores que antecedem o desenvolvimento do agravo à saúde podem levar a um dano e, em conseqüência, esse dano pode representar outros riscos e outros danos sucederem (ALVARENGA, 1986). As relações entre riscos e danos perinatais, neonatais e nas diferentes fases do crescimento da criança, têm implicações para o seguimento. Ao voltarem para casa após a alta hospitalar, as crianças pré-termo e de baixo peso continuarão expostas e vivendo nas condições que desencadearam esses problemas, que são condições de risco para essas crianças que já têm riscos biológicos e que, por sua vez, são frutos de riscos sociais. O seguimento, então, é visualizado no processo de crescimento e desenvolvimento tentando quebrar os elos entre riscos e danos e reduzir vulnerabilidades.

O conceito de risco trouxe para a área da saúde a discussão das diferenças de classes sociais, separando na população aqueles indivíduos, famílias e comunidades com maior probabilidade para determinados danos, passando a revelar de forma mais explícita as possibilidades de articulação entre os níveis políticos, econômico, ideológico e técnico, aparecendo, de certa forma, questões relacionadas ao impasse e inadequação das políticas para atender as necessidades de saúde da população e sobre os altos custos da assistência (MARQUES, 1984).

Ao Estado tem sido colocado o desafio da reconstrução de modelos de atenção à saúde que sejam capazes de detectar necessidades a médio e longo prazo, garantindo o desenvolvimento danação com igualdade e justiça. A intervenção do Estado na área social, tradicionalmente, tem se 
caracterizado pela descontinuidade dos programas e políticas sociais, pela diversidade e superposição de ações e pela instabilidade das suas fontes de receita, havendo necessidade de mudança desse perfil (COHN, 1995). Em termos mundiais, no panorama geral da saúde, as políticas dirigidas ao setor saúde têm grande preocupação com os custos, presentes nos discursos do governo e de agências financeiras internacionais, enfatizando uma política social de cunho neoliberal, com o Estado assumindo pequena parte dos problemas sociais, desfigurando sua função de regulador e prestador de serviços de saúde (COHN, 1995; BANCO MUNDIAL, 1991). As políticas sociais estão determinadas, por um lado, pelos problemas sociais e, por outro, estão limitadas pela estrutura e funcionamento do setor público, particularmente pelos mecanismos de financiamento, em que uma ampla restrição afeta a própria situação social, não atendendo condizentemente as demandas, e aprofundando ainda mais as condições precárias de grupos carentes, os quais dependem dos programas públicos para sobrevivência (SOARES, 1995).

Na presente investigação, compreende-se que os fatores predisponentes à prematuridade e ao baixo peso ao nascer e as relações entre as famílias e os serviços de saúde estão inseridos em um conjunto de determinantes sociais, políticos e econômicos, os quais guardam características marcantes de contradições e conflitos.

Por outro lado, o seguimento de crianças pré-termo e de baixo peso, dentro de um processo de crescimento e desenvolvimento saudável, implica em medidas promocionais, preventivas e de tratamento, ou seja, em vários cuidados à saúde. Além da importância do diagnóstico e da terapêutica, visando a redução da morbimortalidade, a criança e a família necessitam de cuidados básicos, qualquer que seja a fragilidade ou o dano, requerendo atenção profissional para estar junto no cuidar. Repensando a complexidade do cuidado às crianças pré-termo e de baixo peso ao nascer, destacamos o papel da enfermagem. A assistência e o cuidado de enfermagem são consideradas ações que utilizam um saber advindo de outras ciências e de uma síntese produzida pela própria enfermagem para apreender o objeto da saúde naquilo que diz respeito ao seu campo específico para atender necessidades sociais, ou seja, a promoção da saúde, prevenção de doenças e a recuperação do indivíduo ou o controle da saúde da população (ALMEIDA \& ROCHA, 1997). Na enfermagem, o processo de trabalho está voltado para atender uma necessidade de ser cuidado. Cuidar significa assistir o ser humano em suas necessidades básicas, dentro de um caráter uni- versal do cuidado. Entretanto, na prática, ele se apresenta de forma contextual e, portanto, é variável, depende de relações que se estabelecem no processo de assistência. O processo de cuidar implica em aspectos técnico-científicos, biológicos, afetivos, sociais, políticos (ROCHA \& ALMEIDA, 1998).

Neste estudo, destacamos o cuidado domiciliar como parte dos cuidados de enfermagem no seguimento de crianças pré-termo e de baixo peso, entendendo-o como uma prática de saúde dentro da rede de atenção à saúde. Assim sendo, consideramos que apreender os cuidados domiciliares prestados a essas crianças no seguimento após a alta hospitalar oferece possibilidades de conhecer, qualitativamente, como a criança está sendo cuidada no domicílio, quais as ações para prevenção de riscos e danos no processo de crescimento e desenvolvimento de crianças de risco, quais as relações que se estabelecem com os serviços de saúde, e como a enfermagem pode intervir, estabelecendo um conjunto de cuidados com a mãe, que promova a saúde da criança e a tranqüilidade e segurança da mãe.

\section{O CONTEXTO OPERACIONAL DA PESQUISA}

O desenvolvimento deste estudo ocorreu no município de Ribeirão Preto, localizado na região nordeste do Estado de São Paulo. Ribeirão Preto possui, atualmente, onze hospitais, sendo que dez atendem recém-nascidos, entre outras especialidades, e uma rede básica com 30 Unidades Básicas de Saúde, distribuídas em diferentes bairros da cidade. No tocante aos dados sobre nascimentos ocorridos no município de Ribeirão Preto, depreende-se que 99,9\% dos partos são hospitalares (RIBEIRÃO PRETO, 1996). Na distribuição do peso ao nascimento, os recém-nascidos considerados de peso normal perfazem 86,4\% dos partos, e quanto ao Baixo Peso (peso < 2500g) o índice, em 1997, foi de 10,2\%, sendo 1,5\% abaixo de 1500 g, classificados como Muito Baixo Peso. Em relação às gestações de 37 a 41 - semanas completas estavam em torno de $87 \%$ e a prematuridade, gestações antes de completar 37 semanas, em 1997, teve um índice de 8,9\% (RIBEIRÃO PRETO, 1997).

Para a realização da coleta de dados empíricos partimos de uma fase exploratória em que foram selecionados os hospitais do município baseados nos seguintes critérios de inclusão: instituições com grande número de nascimentos de crianças pré-termo e de baixo peso, englobando aquelas de natureza privada e pública. Aten- 
deram a esses critérios quatro hospitais e suas unidades de internação neonatal foram visitadas na tentativa de dialogar com algumas mães que ali estivessem presentes e profissionais de enfermagem, assim como averiguar quais atividades de seguimento pós-alta que essas instituições realizavam. Assim, a partir desse primeiro contato, selecionamos dois hospitais que, pelas evidências empíricas, eram plenos de situações de observação, contatos com mães, seguimento ambulatorial, convênio de saúde, proporcionando um conjunto de informações referentes ao tema do cuidado ao prematuro e ao recém-nascido de baixo peso, objeto desta pesquisa. Um dos hospitais escolhidos foi um hospital público estadual que, além de prestar assistência à população em geral, desenvolve atividades de formação e capacitação de recursos humanos em saúde e de pesquisa, é uma instituição de grande complexidade. O outro hospital é privado, de médio porte, sem vínculo com o Sistema Unico de Saúde (SUS), atende pacientes particulares e de convênios privados de saúde.

A partir da escolha dos hospitais e aprovação do projeto de pesquisa em Comitê de Normas Éticas, iniciamos uma série de visitas semanais aos dois hospitais, realizando o levantamento de dados em prontuários, selecionando crianças com peso de nascimento inferior a 2500 g, idade gestacional menor que 37 semanas e residentes em Ribeirão Preto, observando e dialogando com mães e profissionais de enfermagem, buscando apreender as características dos bebês e mães para comporem os sujeitos desta investigação.

Nessas visitas hospitalares estabelecemos contato inicial com as mães e alguns pais, explicando os objetivos da pesquisa, solicitando sua participação e consentimento, garantindo o sigilo dos dados coletados. Foram selecionadas onze mães e doze crianças, porque houve um caso de gêmeas. Planejamos quatro visitas domiciliares para cada mãe e criança, com intervalo de uma semana entre as visitas. No total foram 44 (quarenta e quatro) visitas realizadas a 11 mães no primeiro mês após a alta das crianças. As razões para delimitarmos o número de visitas, seu intervalo e a duração do seguimento foram de ordem econômica, relacionadas a transporte e tempo.

Neste estudo, o objeto central de interesse, - os cuidados domiciliares prestados durante o seguimento de crianças pré-termo e de baixo peso ao nascer -, não teve a finalidade de estudar a variedade de possíveis seguimentos e sim tentar caracterizar esse cuidado como ele se dá. Procuramos compreender em que se constitui cuidar dessas crianças no período do primeiro mês após a alta hospitalar, as implicações desse cuidado no domicílio, direcionando para poucos casos. Op- tamos pelo desenvolvimento de um projeto de seguimento caracterizando os cuidados de enfermagem, através da observação de algumas crianças, fazendo um recorte temporal, excluindo o interesse por uma amostra que pudesse ser generalizada. Assim sendo, a escolha das mães e crianças esteve permeada de intencionalidade, visando conhecer e refletir sobre os cuidados, o modo de vida da mãe, da criança, suas necessidades, formas de interação, abordando o seguimento de forma particularizada.

A coleta de dados, através de visitas domiciliares, propiciou um contato com a clientela fora dos serviços de saúde, constituindo uma fonte rica e importante de dados para a compreensão da vida cotidiana de mães e bebês. Essas visitas foram realizadas de forma a mesclar a observação, relatos orais e o próprio cuidado, assim como a consulta aos prontuários. Às vezes, um aspecto era mais abordado em uma visita do que em outra, mas sempre partindo de como a criança e a mãe estavam, tendo por referência a semana anterior.

A consulta aos prontuários hospitalares foi a primeira fonte de informações para coleta dos seguintes dados dos bebês e das mães: consultas no pré-natal, número de filhos, idade materna, paridade, intercorrências durante a gestação e o parto, idade gestacional, data e peso de nascimento, duração da internação e convênio de saúde. A observação não foi totalmente estruturada a priori. A estratégia adotada, através de visitas domiciliares, objetivou uma observação livre, para tentar captar a realidade empírica (LUDKE \& ANDRÉ, 1986; MINAYO, 1994). Tivemos como pontos básicos a observação da criança; observação das atitudes da mãe e cuidados relacionados ao bebê durante amamentação, higiene corporal, alimentação, uso de medicações, relacionamento familiar; e observação do ambiente. Quanto aos relatos orais, partíamos da pergunta norteadora: "Como tem sido cuidar do bebê desde que ele nasceu?” e buscávamos incentivar a descrição de situações do cotidiano no cuidado à saúde dos bebês e suas relações com a família, trabalho, escolaridade, renda familiar e os serviços de saúde. Permeando a observação e os relatos orais também estavam presentes as intervenções. Neste estudo, a intervenção foi planejada, fazendo parte do projeto desenvolver o cuidado da criança com a mãe, construindo, através da pesquisa, um conjunto de intervenções de enfermagem necessárias ao acompanhamento de crianças pré-termo e de baixo peso. As informações coletadas foram registradas em um diário de campo, contendo anotações sobre as observações, relatos das mães e intervenções, além de anotações reflexivas dos pesquisadores. 


\section{CARACTERIZAÇÃO DAS CRIANÇAS E MÃES ESTUDADAS}

Os doze bebês observados nasceram no período de julho a novembro de 1997, sendo cinco do sexo feminino e sete do masculino. O peso de nascimento variou de $150 \mathrm{~g}$ a $238 \mathrm{~g}$, sendo que cinco crianças foram classificadas como de Muito Baixo Peso. Em relação à idade gestacional, houve amplitude de variação de 29 a 39 semanas, sendo uma criança classificada dentro da prematuridade extrema (abaixo de 30 semanas de gestação), oito como pré-termo moderado (entre 30 e 34 semanas), uma como pré.-termo limítrofe (entre 35 e 36 semanas). No caso de uma das criança, essa informação não se encontrava no prontuário, mas as evidências físicas e cuidados registrados retratavam a prematuridade. Outra criança era de baixo peso e a termo, com 39 semanas de gestação e, embora não classificada como pré-termo, foi incluida nos estudos pelas características maternas, importantes para a questão dos deteminantes sociais do baixo peso ao nascer. A duração da internação esteve associada a intercorrências clínicas e à evolução de cada criança, apresentando uma variação de 6 a 72 dias. Das doze crianças observadas, dez passaram pela Unidade de Terapia intensiva Neonatal, sete foram hospitalizadas em serviço público e cinco em serviço particular, houve dois casos de transferência do hospital privado para o público, em decorrência de acertos sobre a internação a cargo de convênios de saúde.

Quanto às características maternas, as idades limites foram 16 e 41 anos,sendo três abaixo de 20 anos e três acima de 30, seis mães eram primigestas e cinco multigestas, todas tiveram acesso ao pré-natal, embora uma delas tenha sido considerada irregular pelo início tardio, a partir do sétimo mês de gestação, com apenas uma consulta antes de o bebê nascer. Em relação ao tipo de parto, nove foram cesária, um normal e um fórceps. A escolaridade materna variou de 3 a 15 anos de estudo, sendo que seis mães não completaram o ensino fundamental. Sobre a ocupação materna, a maioria trabalha no lar, somente duas mães tinham vínculo empregaticio, porém durante a investigação estavam em licença-maternidade. A renda familiar variou de 200 a 300 reais, para um número de moradores no domicílio de três a sete membros. O rendimento mensal provinha do trabalho do pai e as mães adolescentes recebiam ajuda financeira dos familiares. Em relação à moradia, das onze famílias estudadas, duas moravam em favelas e nove em casas de alvenaria, localizadas em bairros periféricos do município de Ribeirão Preto.
No tocante às mães e crianças investigadas, entendemos que as famílias que estavam residindo em favelas ou em situação semelhante estavam passando por condições mais difíceis, as outras famílias pareciam não apresentar condições adversas, embora não fossem homogêneas quanto à escolaridade, rendimentos mensais, número de membros na família e acesso aos serviços de saúde.

Entre os fatores de risco mencionados na literatura, destacamos o baixo peso ao nascer, a idade e escolaridade materna, gemelaridade, acesso ao pré-natal e, em alguns casos, residência em microárea de risco, características essas que aumentam a probabilidade de morbidade perinatal e infantil, associados à prematuridade ou intercorrências como diarréias, verminoses, infecções respiratórias. Observamos que para a mãe mais jovem, primigesta, com baixa escolaridade ou em condições de moradia menos satisfatórias, questões envolvendo noções básicas de higiene, medidas de prevenção, observação c e sinais e sintomas na criança, comunicação, ambiente desorganizado, entre outras, refletiam-se no cuidado cotidiano da criança, requerendò suporte profissional, um estar junto auxiliando e orientando. As mães com melhores condições sócioeconômicas, acesso aos bens e informações, maior conforto e espaço doméstico, também apresentavam insegurança quanto aos cuidados básicos, e a presença do profissional facilitou ou aperfeiçoou suas ações com a criança.

\section{CUIDADOS DE ENFERMAGEM NO SEGUIMENTO DOMICILIAR DE CRIANÇAS PRÉ-TERMO E DE BAIXO PESO AO NASCER}

A análise do conjunto de informações registradas sobre as observações, relatos orais e intervenções possibilitou a apreensão de dois temas: hospitalização e vínculo, cuidados de saúde infantil no domicílio, os quais permitiram construir aspectos fundamentais para o seguimento de enfermagem às crianças pré-termo e de baixo peso através de visitas domiciliares.

Embora o enfoque da investigação tenha sido o primeiro mes após a alta hospitalar da criança, foi muito significativo o período de hospitalização conforme apresentado nos relatos das mães, configurando os primeiros aspectos do seguimento. Ao mesmo tempo que estávamos junto com a mãe no cuidado e na observação da criança, essas informações retrospectivas surgiram, porém não se tratava de histórias de vida de longa data mas de histórias recentes. Os relatos dessa experiên- 
cia recém-vivida continham aspectos precisos por serem próximos e estarem repercutindo na vivência da mãe com a criança, extremamente importante para o relacionamento com o filho e para todo o processo de crescimento e desenvolvimento infantil. O seguimento domiciliar tem início ao conhecermos a vivência da mãe sobre o processo de hospitalização do filho, devendo priorizar os significados que ela atribui a esse período, as questões da dor e do sofrimento pela separação do filho, a necessidade materna de querer ficar com o filho durante a internação, conhecer sua gravidade e riscos. As mães se colocaram quanto ao acesso ao serviço e à sua participação, ou não, nos cuidados com o bebê, sendo a amamentação o grande elo de ligação, assim como algumas mencionaram o apoio do marido na amamentação e nas visitas ao filho. Ao relatarem sua participação na assistência ao bebê, emergiam dois aspectos: um quanto aos conhecimentos assim adquiridos, muitas vezes possibilitado pela observação do trabalho de enfermagem; outro, foi seu envolvimento pessoal no cuidado, desenvolvendo autoconfiança para o período após a alta. Deste modo, quando as mães buscam ajudar nos cuidados à criança pré-termo e de baixo peso, procuram aprender a maneira de oferecer tais cuidados, ao mesmo tempo que mantêm vigilância quanto ao progresso clínico, tentam dividir um pouco o trabalho com os profissionais que cuidam do bebê, buscando informações, recursos para avaliação, tratamento e suporte (BARNETT et al., 1970; KLAUS \& KENNELL, 1970; NARAYANAN et al., 1991; BARRAM et al., 1992; McKIM, 1993; MAY, 1997). O suporte profissional, em particular o de enfermagem, está vinculado aos cuidados básicos, cuidados higiênicos, alimentares, terapêuticas, conforto, bem como o envolvimento emocional e de orientações educativas.

Algumas mães vivenciaram esse período com limites oriundos de dificuldades financeiras ou de restrições devido a rotinas hospitalares, com os limites para os horários de visitas. A falta de dinheiro para o transporte até o hospital, para as visitas e para os retornos agendados no seguimento ambulatorial, foi igualmente colocado como problema por autores que estudaram essas questões (McLOUGHLIN, 1995; ZAHR, 1994). O baixo nível socioeconômico interfere nas ações dos pais, eles desejam cuidar mais e melhor dos filhos, e o fato de não poderem realizar isso devido à situação financeira, traz frustração e estresse (BRADLEY et al., 1994; ZAVASCHI et al., 1998). Compreendemos que os aspectos apontados estão ligados à relação de apego mãe-filho, entendendo-a não como uma relação idealizada ou que ocorre naturalmente, mas permeada pelas preo- cupações da família, pelas distâncias, pelas visitas esporádicas, pela participação do pai e dos enfermeiros que interferiram nessa relação. Depreendemos que o serviço hospitalar está em processo de transformação quanto à participação da mãe na interação com a criança hospitalizada; essa relação, por sua vez, é construída, e pode ser ainda mais aprimorada quanto mais atentos a ela estivermos. Nesse sentido, entendemos que a enfermagem tem um papel fundamental nessa construção, oferecendo possibilidades para a mãe e a família, ajudando, orientando, avaliando e dando atenção para a sua participaçao na assistência, visando implementar, deste modo, ações de cuidado para o pleno crescimento e desenvolvimento infantil.

Em relação aos cuidados de saúde infantil no domicílio durante o primeiro mês após a alta hospitalar, exploramos o que era perceptível e relevante para esse período em termos de cuidados básicos domiciliares, podendo conter indicativos para o seguimento durante a infância. Nas visitas domiciliares semanais foram realizadas observações e intervenções relacionadas aos aspectos físicos, alimentares eterapêuticos, enfatizando a interação com as mães. Realizamos exame físico das crianças, priorizando a observação da aparência geral, condições da pele e higiene, fontanelas, olhos, nariz, boca, padrão respiratório, abdome, genitais, eliminações e presença de reflexos. Não foi prioridade a mensuração do peso, estatura ou perímetro cefálico, embora consideradas medidas de extrema importância para avaliações do crescimento infantil. Os dados estavam presentes na história da criança, relatada pela mãe, e o ganho de peso foi um aspecto muito enfatizado. Conhecer o peso da criança e acompanhar quase que semanalmente o crescimento tem o significado, para as mães, de um envolvimento com a saúde dos filhos, expressando um sucesso ou fracasso do desempenho materno nos cuidados realizados no domicílio. Observamos que as mães manifestavam muita alegria ao nos contar sobre o ganho de peso do bebê de uma semana para outra, ou tristeza e desânimo se não ocorria bom ganho ponderal. No período investigado, pareceu-nos que as mães ficaram mais ansiosas em relação ao baixo peso do bebê do que quanto à prematuridade. Entendendo que o baixo peso ao nascimento e a prematuridade são situações muito próximas, para elas, o peso nesse primeiro mes após a alta é mais visível, e o ganho de peso constitui o principal parâmetro do crescimento e desenvolvimento da criança As doze crianças observadas apresentaram boas condições de evolução nas quatro visitas domiciliares realizadas para cada urna. 
O exame das crianças era realizado partindo das colocações da mãe que, enquanto contava como a criança estava passando, já ia retirando a roupinha ou para o banho de banheira ou ante uma solicitação nossa. Era o momento para a observação da coloração e integridade da pele, para a avaliação do padrão respiratório, de aspectos ligados a termorregulação, entre outros. Três crianças com características relacionadas aos quadros clínicos do período neonatal, broncodisplasia pulmonar, refluxo gastroesofágico e ileostomia após enterocolite necrosante, fizeram parte de um seguimento domiciliar e ambulatorial mais específico. Durante as visitas tivemos várias oportunidades para esclarecimento sobre as condições de saúde dos bebês, revendo e incentivando a amamentação, higiene das mamadeiras, conservação e administração de medicações em uso, preparo minucioso e oferecimento mais cuidadoso da alimentação, observação do padrão respiratório, ganho de peso, prevenção de infecções, estimulações e interação mãe-filho. As mães vivenciaram momentos de dúvidas, se suas condutas nos cuidados diários podiam ser adequadas ou, às vezes, gerar alguma situação de risco, seja no preparo de uma mamadeira, na sucção ineficiente do peito, na avaliação da respiração, no preparo de medicações ou qualquer outro procedimento.

Nessa caracterização da assistência domiciliar à saúde da criança, em que apreendemos os aspectos físicos, alimentares e terapêuticos, destacamos os cuidados básicos de enfermagem voltados para as necessidades básicas de conforto e segurança, higiene corporal e proteção da pele, respiração, temperatura, alimentação e hidratação, medicações, sono, repouso e vestuário, aspectos esses que configuram a universalidade do cuidar em enfermagem. No entanto, também apreendemos, no processo de assistência, variações conforme o ambiente que vive a criança, a interação que se estabeleceu entre a mãe e a criança e as condições sociais da família. Por um lado, a capacitação técnica do enfermeiro pode dar sustentação para o entendimento dos problemas de saúde, desencadeando intervenções, propondo soluções cotidianas, estabelecendo comunicações com a rede de serviços, promovendo educação em saúde e prevenindo danos para crianças prétermo e de baixo peso e, por outro lado, articulado a tudo isso, também pode constituir uma prática permeada de ajuda, afetividade, sensibilidade e intersubjetividade.

O processo de cuidar, englobando as dimensões técnica, política e de interação, implica trabalhar as necessidades da criança com o envolvimento da família, o enfermeiro interagindo com a criança , sua família, os serviços de saúde e a comunidade. Assim, a assistência prevê uma interação facilitadora para o crescimento e desenvolvimento infantil e a superação de dificuldades inerentes ao processo saúde-doença, com elaboração compartilhada de um conjunto de cuidados, considerando os conhecimentos, experiências e habilidades do enfermeiro, da criança e da família, atentando para o contexto social, sistema de hábitos e valores da criança e familiares (ANGELO \& VERÍSSIMO, 1996).

Para BRADLEY et al. (1994), o cuidado é permeado por fatores protetores e regula o crescimento e desenvolvimento em cinco caminhos básicos: ao prover sustento, alimento, promove a saúde e a integridade biológica; ao prover estimulação, promove funções cognitivas e adaptativas; ao prover suporte, promove a integridade emocional; ao prover estruturas, maximiza uma contribuição positiva de sustento, estimulação e suporte garantindo uma adequação entre o que o ambiente tem de recursos e o que o organismo necessita; e ao prover vigilância e continuidade, aumenta a probabilidade colocada no organismo para um desenvolvimento estruturado. Nessa perspectiva, o cuidado domiciliar tem como finalidade a promoção do bem-estar da criança e de sua família. Tal finalidade fundamenta-se em ações elaboradas a partir da prevenção de danos, de identificação de riscos e da assistência à criança para um adequado crescimento e desenvolvimento.

\section{CONSIDERA ÇOES FINAIS}

A presente investigação possibilitou apreender alguns elementos importantes que compõem os cuidados de enfermagem no seguimento domiciliar de crianças pré-termo e de baixo peso ao nascer. Realizado no período pós-alta imediato, envolve cuidar da criança, cuidar da mãe, articulações com os serviços de saúde, levantamento de dados sobre o ambiente e preparo técnico-científico do profissional de enfermagem.

As ações de enfermagem no domicílio perfazem cuidados técnico-científicos baseados em conhecimentos clínicos, de prevenção, de higiene, de alimentação, do efeito de medicações, sobre imunização, sobre o controle de retornos, entre outros, configurando aspectos gerais e específicos da atenção à saúde da criança. O seguimento domiciliar pela enfermagem requer, além destes, uma outra visão sobre visita domiciliar, não podendo ser esta apenas informativa ou assistencial. $\mathrm{O}$ trabalho de enfermagem nesse âmbito implica em uma nova visão do cuidado que envolve o estar no domicílio com a mãe. É um atendimento que deve prever um maior tem- 
po de visita e um maior número de retornos à casa, para poder acompanhar, estar presente participando e tentando compreender, os vários aspectos vivenciados pela mãe, pela criança e pela família. Na articulação dos serviços de saúde é importante o papel da enfermagem que atua nos berçários e na rede básica de saúde para estabelecer comunicações interinstitucionais. É fundamental levantar informações sobre o contexto familiar e social da criança que está hospitalizada, retornando-as para a assistência ali prestada e também para o processo de seguimento pós-alta. São importantes o diagnóstico da situação e as conexões e retroalimentação entre os serviços de saúde, repassando e integrando informações e intervenções. O avanço nas políticas sociais pode trazer muitos beneficios às famílias, com destaque para a área da saúde e de outros setores sociais, com o suporte profissional favorecendo o bem estar dos pais, detectando carências, identificando recursos da comunidade, intensificando a atenção à saúde nas unidades básicas de saúde, em visitas domicilia- res, proporcionando melhoria da qualidade de vida.

Este estudo do seguimento domiciliar de crianças pré-termo e de baixo peso ao nascer mostrou que, uma parte da atenção à saúde, os cuidados básicos de enfermagem -, pode ocorrer em intervenções em conjunto com a mãe, permitindo precocidade e continuidade no acompanhamento da criança, soluções compartilhadas, percepção da situação de saúde da criança, adesão a determinadas terapêuticas, prevenção de riscos e danos, encaminhamentos, interação profissionalmãe-criança. Mostrou, igualmente, a construção de uma prática de saúde com relações de proximidade, de acolhimento, de interação facilitadora e de superação de dificuldades, proporcionando suporte à mãe e auxiliando em sua autoconfiança para quanto a um adequado crescimento e desenvolvimento da criança. Cabe mencionar que essas crianças continuam sendo acompanhadas como parte de outro estudo, para além do primeiro mes após a alta hospitalar.

\begin{abstract}
The mursing care given to preterm and low birth weight infante advanced a lot in recent years with the teclmology increment and lhe decline in infant death rales. This caused lhe survival of a higher number of children and triggered discussions about the quality of life, lhe care needs and lhe follow-up over extended periods of time. The aim of this investigation is to identify the home care given to preterm and low birth weight infants, so as to characterize the follow-up during the first month after discharge from hospital. The methodological focus brings approaches from epidemiology, nursing care and health policies. The empirical study on home care, based on data consultation in the hospital files, participatory observation and mothers' oral reports at home, presents a characterization of the analysed children and mothers, hospitalization and attachment and the infant's health care at home. Following this configuration we point to aspects of these children's follow-up through home visiting, composing basic elements of nursing care. Home follow-up involves taking care of the child according to its clinical needs, risks and damages prevention, alimentation, hygiene, use of medication, sensorial stimulation, among others. It also involves the care given by the mother by its side, listening to it, reviving expenences of the child's hospitalization process, tahng care togeffier wiffi ffie child, giving education in healffi, comffirt, self-assurance. At the same time, home care implies surveying offier family needs, raising community resources, establishing am integration of information and mtervention between the health services and the support services.
\end{abstract}

Key-words: preterm; low birffi weight; follow-up; nursmg. 


\section{REFERÊNCIAS BIBLIOGRÁFICAS}

ALBERMAN, E.; EVANS, S.J.W. Epidemiologia da prematuridade: etiologia, ffequência e prognóstico. In: NESTLÉ Nutrifion Services, O Prematuro. Anais Nestlé, 44: 5-24, 1992.

ALMEIDA, M.C.P; ROCHA, S.M.M. (Orgs.). O trabalho de enfermagem. Sào Paulo, Cortez, 1997.

ALVARENGA, A.T O enfoque de risco. In: SEMINÁRIO ESTADUAL DE SAUDE DA CRIANÇA 11., Sào Paulo, 1986. Anais. Sào Paulo, Secretaria da Saúde/Instituto de Saúde, 1986. p.1924.

AMON, E.; ANDERSON, G.D.; SIBAI, B.M.; MABIE, W.C. Factors responsable for preterm delivery of the immature newborn infant (=lOOOg), AMJ ObstetGynecol ,156(5): 11431148,1987.

ANGELO, M.; VERÍSSIMO, M.D.L.O.R. O papel de enfermeira centrado na criamça e na familia. In: SIGAUD, C.H.S.; VER^íSSIMO, M.D.L.O.R. (Orgs.). Enfermagem pediátrica: o cuidado de enfermagem à criança e ao adolescente. São Paulo, EPU, 1996. p.89-96.

AVERY, G.B. Neonatology: perspective in ffie mid 1990s. In: AVERY, G.B.; FLETCHER, M.A.; McDONALD, M.G. Neonatology: pathophysiology and management of newborn. 4 ed. Philadelphia, J.B. Lippmcot Compamy, 1994.

BANCO MUNDIAL. Brasil - nova desaf o à saúde do adulto. Novas realidades economicas, demográficas e epidemiológicas. Série de estndos do Bamco Mundial sobre países. Washington, D.C., 1991. 134p.

BARBOSA, N.M.M.; CABRAL, A.H.L.; SILVA, O.PV; MOSZKWICZ, B. Follow-up do bebê de risco. Temas de pediatria-Nestlé, 53, 1993.

BARNETT, C.R; LEIDERMAN, P H.; GROBSTEIN, R.; KLAUS, M. Neonatal separation: ffie matemal side of mteractional deprivation. Pediatrics, 45(2): 197-205, 1970.

BARRATT, M.S.; ROACH, M.A.; LEAVITT, L.A. Early channels of moffiers infantil communication: preterm and term infants. J. Child Psychol.

BRADLEY, R.H.; WHITESIDE, L.; MUNDFROM, D.l.; CASEY, PH.; KELLEHER, K.l.; POPE, S.K. Early indications of resilience and ffieir relation to experiences in the home enviromments of low birffiweight, premature chidren living in poverty. Child Development, 65(2): 346-360, 1994.

COHN, A. Políticas sociais e pobreza no Brasil. Planejamento e Políficas Públicas.Brasilia, 12: p. 1-18, jul./dez.1995.

FUNDAÇÃO INSTITUTO BRASILEIRO DE GEOGRAFIA E ESTAT^^́́STICA. Crianças e adolescentes: indicadores sociais. Rio de Janeiro, IBGE, 1992.

KLAUS, M.H.; KENNELL, J.H. Moffiers separated ffom thenr newbom infants. Pediatrics Clinics of North America, 17(4): 1015-1037,1970.

LUDKE, M.; ANDRÉ, M.S.D.A. Pesquisa em educação: abordagens qualitativas. São Paulo, EPV, 1986.

MARQUES, M.B. A atenção materno-infamtil como prioridade política. In: GUIMARÃES, R. (Org.). Saúde e medicina no Brasil: contribuição para um debate. Rio de Jameiro, Graal, 1984.

MAY, K.M. Searching for normalcy: moffiers' caregiving for low birth weight infants. Pediatric. Nurs.,23(1): 17-20, 1997.

McKIN, F M. The information amd support needs for moffiers of premature infamts. J Pediatr Nurs., 8(4): 233-244,1993.

McLOUGHLIN, A. Formal and informal support mothers who have had a baby in a neonatal intensive care unit. Mamchester, 1995. [Thesis of Doctorate - School of Nursing Studies, University of Manchester]

MINAYO, M.C.S. O desaf o do conhecimento: pesquisa qualitativa em saúde. 3ed. São Paulo Rio de Janeiro, Hucitec/Abrasco, 1994.

MONTEIRO, C.A.; ISHII, M.; BENICIO, M.H.d'A; REA, M.F A distribuição do peso ao nascer no município de Sào Paulo, Brasil. Rev. Saúde Públic., S. Paulo, 14(2): 161$172,1980$.

NARAYANAN, 1. et ai. Maternal participafion in ffie care of ffie high risk mfamt: follow-up evolution.

PENALVA, O. Seguimento de bebas de alto-risco. In: Curso Nestlé de Atualização em Pediatria, s3., Manaus, 1996. Anais. Manaus, Serviço de Informação Científica Nestié, 1996, p. $135-139$.

RIBEIRÃO PRETO. Sistema de informações sobre nascidos vivos. Ribeirão Preto, Secretaria Municipal da Saúde, Departamento de Informática, 1996. [mimeografado] 
RIBEIRÃO PRETO. Sistema de informações sobre nascidos vivos. Ribeirão Preto, SecretariaMunicipal da Saúde, Departamento de Informática, 1997. [mimeografado]

ROCHA, S.M.M.; ALMEIDA, M.C.P. O processo de trabalho da enfermagem em saúde coietiva e a intersetorialidade. In: Encontro Estadual de Enfermagem em Saúde Coietiva, I, Canoas- RS, 1998. ProgramaeResumos. Canoas- RioG^IandedoSul, 1998.

SCHAFFER, A.J., AVERY, M.E. Doenças do recémnascido. 4aed. Rio de Janeiro, Interamericana, 1979.

SOARES, L.T. Ajuste neoliberal e desajuste social na América Latina. Campinas, 1995. Tese ( Doutorado) - Instituto de Economia da Universidade Estadual de Campinas.
WELDT, E; et ai. Seguimento de ninõs com peso ai nacer inferior a $1500 \mathrm{~g}$. Rev. ChiL Pediatr., 60(3): 129-134,1989.

ZAHR, L. et ai. An integrative research review of intervention studies with prematuro infante from disadvantaged backgrounds. Mat-Child

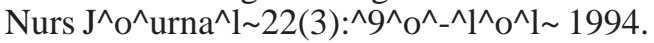

ZAVASCHI, M.L.S. et al. A influência de aspectos socioeconômicos desfavoráveis sobre a interação mãebebê.RevistaABP-APAL, 20(2): 66-70, 1998.

Recebido em 08/03/2000

Aprovado em 31/06/2000 